\title{
A Study on the Applications of Laplace Transformation
}

\author{
Gagan Gaba \\ Department of Mathematics \\ Email id : gagangaba100@gmail.com \\ Chandigarh University ,Mohali, Punjab
}

\begin{abstract}
Mathematics plays an important role in our everyday life. Laplace transform is one of the important tools which is used by researchers to find the solutions of various real life problems modeled into differential equations or simultaneous differential equations or Integral equations. In this paper, we are going to study the details on lapace transform,its properties and "Applications of Laplace Transform in Various Fields". Various uses of Laplace Transforms in the research problems have been highlighted. Detailed applications of Laplace Transform have been discussed.
\end{abstract}

\section{INTRODUCTION}

The main purpose of the Laplace Transformation is that it coverts system of equations or differential equations or integral equations into a simpler form so that we can get the solution in an easy way. There are various problems that are really difficult to solve or algebraically very unique . Laplace transformation plays a vital role in finding the solution of complex integral functions. In general the method of Laplace Transformation is required to find $n$th order Linear Differential Equations with constant coefficients.

Laplace transformation is an effective tool for examining circuits with sinusoidal or nonsinusoidal data sources. The vast choice of applications make the method of laplace transformation a big asset to study the attributes of a large number of problems (Hailongchen et al, 2015). Generally, we use laplace transform for solving differential equations with higher orders. It has a huge number of applications in the field of mathematics, engineering and applied sciences. [1]. Laplace transform is generally utilized strategy which tackles with higher order differential equations. It is utilized for adjusting indispensable, circuit frameworks, mechanical frameworks, aeronautics frameworks, picture handling, etc.

\section{PRELIMINARIES}

Laplace Transform: The Laplace transformation are the mathematics operations that convert a particular function from time domain to complex frequency domain. Laplace transforms play a vital role in solving differential equations with boundary values without finding the values of arbitrary constants and the genral solution making it easier than other methods, 


$$
f(t) \rightarrow F(s)
$$

where(t) represent time and (s) represent complex frequency

Laplace transform definition is given by

$\mathrm{F}(S)=\int_{0}^{\infty} f(t) e^{-s t} \mathrm{dt}$

Various properties of Laplace transforms

1. Linearity theory

2. Laplace transform for derivative

3. Laplace transform for integral

4. Initial value theorem

5. Final value theorem

Linearity Theory : The Laplace transform in actual it's integration operation that be Linear operation undergo to superposition method.

$$
L\{a f(t)+b g(t)\}=a L\{f(t)\}+b L\{g(t)
$$

$$
\text { Example } \begin{aligned}
: L \sinh (t)\}= & L\left\{\frac{1}{2} e^{t}-\frac{1}{2} e^{-t}\right\}=\frac{1}{2} L\left\{e^{t}\right\}-\frac{1}{2} L\left\{e^{-t}\right\} \\
& =\frac{1}{2}\left\{\frac{1}{(s-1)}-\frac{1}{(s+1)}\right\} \\
& =\frac{1}{2}\left\{\frac{(s+1)-(s-1)}{\left(s^{2}-1\right)}\right\}=\frac{1}{\left(s^{2}-1\right)}
\end{aligned}
$$

Laplace For Derivative: For $\mathrm{t} \geq 0$, let $f(t)$ be function which is having an exponential order ' $\mathrm{a}$ ' and is continuous on $[0, \infty)\left(\right.$ so that $\left.\lim _{t \rightarrow \infty} e^{-s t} f(t)=0\right)$. Let $f^{\prime}(t)$ be also continuous and of exponential order ' $a$ ' or piecewise continuous on $[0, \infty)$, then

$\mathrm{L} f^{\prime}(t)=s F(s)-f(0)$ for $\mathrm{s}>\mathrm{a}$, where $\mathrm{Lf}(\mathrm{t})=\mathrm{F}(\mathrm{s})$

Initially we have derivative of time domain then we convert it into algebraic equation in Laplace domain.

Laplace For Integral: The integration theorem includes:

$$
\text { If } L[f(t)]=F(s) \text {, then } L \int_{0}^{t} f(u) d u=\frac{1}{s} F(s)
$$

Evaluation of integral:

$$
\text { If } L[f(t)]=F(s) \text { i.e., } \quad \int_{0}^{\infty} e^{-s t} f(t) d t=F(s)
$$


Taking the limits as $s \rightarrow 0$

$$
\int_{0}^{\infty} f(t) d t=F(0)
$$

By assuming the integral to be convergent..

Initial Value Theorem:This includes

$$
\lim _{t \rightarrow 0}(f(t))=\lim _{s \rightarrow \infty} s L[f(t)]
$$

This theorem is applicable only when we have the power of numerator polynomial less than that of denominator i.e. $F(s)=\frac{1}{s+1}$

Final Value Theorem: It have

$$
\lim _{t \rightarrow \infty} f(t)=\lim _{s \rightarrow 0} s L\{f(t)\}
$$

Increasing exponentials (like $e^{a t}$ where $a$ is a positive number) that goes to $\infty$ as $t$ increases, and the oscillating functions (like sine and cosine that have no final value) are examples for which this theorem can't be used.

\section{TABLE 1 RESULTS}

\begin{tabular}{ccc}
\hline Property & $\mathbf{F ( t )}$ & $\mathbf{F}(\mathbf{s})$ \\
\hline Linearity & $a_{1} f_{1}(t)+a_{2} f_{2}(t)$ & $a_{1} F_{1}(s)+a_{2} F_{2}(s)$ \\
Scaling & $f(a t)$ & $\frac{1}{a} F\left(\frac{s}{a}\right)$ \\
Time Shift & $F(t-a) u(t-a)$ & $e^{-a s} F(s)$ \\
Frequency Shift & $e^{-a t} f(t)$ & $s(s+a) \mathrm{a})$ \\
Time differentiation & $\frac{d f}{d t}$ & $s^{2} F(s)-s f(0)-s f^{\prime}(0)$ \\
$\frac{d^{2} f}{d t^{2}}$ & $s^{3} F(s)-s^{2} f(0)-s f^{\prime}(0)$ \\
$\frac{d^{3} f}{d t^{3}}$ & $s^{n} F(s)-s^{n-1} f(0)$ \\
$\frac{d^{n} f}{d t^{n}}$ & $-s^{n-2} f^{\prime}(0) \ldots . f^{n-1}(0)$ \\
\hline
\end{tabular}




$\begin{array}{ccc}\text { Time Integration } & \int_{0}^{1} f(t) d t & \frac{1}{s} F(s) \\ \text { Frequency differentiation } & \mathrm{tf}(\mathrm{t}) & -\frac{d}{d s} F(s) \\ \text { Frequency Integration } & \frac{f(t)}{t} & \int_{x}^{\infty} F(s) d s \\ \text { Time periodicity } & f(t)=f(t+n T) & \frac{f_{1}(s)}{1-e^{s T}}\end{array}$

Laplace Transform Of Some Elementary Functions

1. $L(1)=\frac{1}{s}$

2. $L\left(t^{n}\right)=\Gamma(\mathrm{n}+1)$ if $\mathrm{n}$ is any real number $>-1$ and $\mathrm{s}>0$ $L\left(t^{n}\right)=\frac{n !}{s^{n+1}}, n=0,1,2, \ldots$.

3. $L\left(e^{a t}\right)=\frac{1}{s-a}$

4. $L(\sin a t)=\frac{a}{s^{2}+a^{2}}$

5. $L($ cosat $)=\frac{s}{s^{2}+a^{2}}$

6. $L(\sinh a t)=\frac{a}{s^{2}-a^{2}}$

7. $L($ coshat $)=\frac{s}{s^{2}-a^{2}}$

\section{INVERSE LAPLACE TRANSMATION}

This type of transform is beneficial and very useful to find the solutions of the differential equations. When we take the Inverse laplace transform to $F(s)$, it gives $f(t)$. In other words it converts function from complex frequency domain to time.

The general Form of Inverse Laplace transformation is ,

If $\mathrm{L}\{\mathrm{f}(\mathrm{t})=\mathrm{F}(\mathrm{s})$, then $\mathrm{f}(\mathrm{t})$ is called inverse laplace tranformation of $\mathrm{F}(\mathrm{s})$ and is written as,

$$
L^{-1}\{F(s)\}=f(t)
$$

Here $L^{-1}$ denotes the inverse Laplace transform

For example, Since $L\left\{e^{2 t}\right\}=\frac{1}{s-2}$, we have $L^{-1}\left\{\frac{1}{s-2}\right\}=e^{2 t}$ 
Properties of Laplace transform are also followed by Inverse Laplace transforms.

1. $L^{-1}\left\{\frac{k}{s}\right\}=k, k$ being constant

2. $L^{-1}\left\{\frac{1}{s-a}\right\}=e^{a t}$

3. $L^{-1}\left\{\frac{1}{s^{n}}\right\}=\frac{t^{n-1}}{(n-1) !}$ if $\mathrm{n}$ is positive integer. Otherwise $=\frac{t^{n-1}}{\Gamma(n)}$

4. $L^{-1}\left\{\frac{1}{(s-a)^{n}}\right\}=e^{a t} \frac{t^{n-1}}{(n-1) !}$

5. $L^{-1}\left\{\frac{1}{s^{2}+a^{2}}\right\}=\frac{1}{a} \sin a t$

6. $L^{-1}\left\{\frac{s}{s^{2}+a^{2}}\right\}=\cos a t$

7. $L^{-1}\left\{\frac{1}{s^{2}-a^{2}}\right\}=\frac{1}{a} \sinh a t$

8. $L^{-1}\left\{\frac{s}{s^{2}-a^{2}}\right\}=\cosh a t$

\section{ADVANTAGES}

In comparison to traditional approaches, Laplace transforms have the following advantages.

1. It provides a comprehensive solution.

2. In the transformed equations, initial conditions are taken into account automatically.

3. Solving differential equations takes a lot less time.

4. Generally, valuable and systematic solution can be find for any problem (philipmicheal, 2009)

\section{APPLICATIONS}

In this portion, we are going to study about the applications of Laplace transforms. The study will help us to understand the applications or uses of this technique in various research problems. The study of various research articles is done. The applications of Laplace transformation techniques and their functionality is being discussed in this paper.

\section{Duplication of impulse response of electric machines:}

Machines that are electric in nature are regularly presented in assortment of structures of wave which create issues in them. The work is performed to duplicate these types of motivation reactions and to give think about them. The work is done to duplicate these motivation reactions and think about them. Metwally, 1999 [2] discussed about three types of strategies to keep a check on transient reaction of electrical machines are examined, these are: (a) approach of state 
space. (b) Method of Laplace Transformation. Laplace Transformations are used to dissect voltage transient.

\section{Non-linear form of large systems:}

Hasan Modirshanechi and Naserpari, 2003 [3] presented and built up another strategy called modular arrangement technique, which addresses non linear framework reaction for even zero contribution to the type of Differential Equations. It helps to determine and addresse the conduct of non-linear unique frameworks utilizing non-linear modular portrayal. For this, Laplace Transformation is used to solve the problem of non-linear Differential Equations.

\section{Back to back high voltage dc converters (three-level) based on $\mathrm{h}$ - bridge converters:}

Displaying of high voltage D.C.(Direct Current) converters dependent on H-connect voltage converters are exhibited by SiriyaSkolthanarat, 2007 [4]. This has more highlights contrasted with two-level Converters. It revises power framework phenomena like force quality, voltage and first swing steadiness. Plans of P.I type compensators are inferred by Laplace Transformations.

\section{Scientific approach for broadband multi electro chemical piezo electric bimporph beams with multi frequency power harvesting:}

Peter Lloyd woodfield, 2015 [5] determined the multi-recurrence reactions of multi-electro substance piezo-electric bimorph radiates are dependent on shut structure limit esteem strategy from solid type of Hamiltonians standard. Likewise talked about the conversion of unused mechanical energy into electrical energy by planning reasonable electro mechanical framework. Laplace transform is utilized to plan new formulae for power reaping multi-recurrence reactions in numerous bimorph light emissions kinds of associations.

Variational Principles for Heat Conduction :-Classical variational standard does not exist for explanatory and exaggerated warmth conduction equations.P. Szymczyk, M. Szymczyk, 2015 [6] clarified and talked about the standards of those equations. In this traditional variational standard is portrayed to models like cattaneo-vernotte model, Jeffrey model, two temperature models to say a couple. Laplace transformations are utilized to determine old style variational standards.

Short-term detailed solutions for the motion of a vibrating cylinder in the stokes regime Laplace transforms:

A fixed Newtonian liquid, an answer for transient rot of snapshot of vibrational chamber is concentrated by Shu-NanLi, Bing-Yang Cao, 2016 [7]. Snapshot of flexible chamber is likewise talked about. In this full articulations for transient terms are determined. It likewise have applications in thickness estimations. Laplace transformation is utilized to infer scientific answers for snapshot of versatile chamber in Newtonian liquid. 


\section{Analysis of structural geology using ground-penetration radar and Laplace transform artificial neutral networks:}

Mikail. F. Lumentat, 2012 [8] depicted another kind of fake neurons and neural organizations. By utilizing these neural organizations and on premise of various kinds of geographical constructions, the design of topographical substance is grouped. Laplace transform is utilized rather than fake loads and in linear enactment capacity of counterfeit neuron.

\section{Sar -image despeckling based on combination of Laplace mixture distribution with local parameters and multiscale edge detection in Laplace transform domain :}

The impact of dot clamor on assignments of programmed data extraction and SAR pictures is concentrated by Hazarika et al., 2016 [9]. Also, this impact is redressed utilizing Laplace Transform Technique. Another and successful strategy is created to SAR picture Despeckling. Another sort of Laplace symmetrical transform (LOT) is proposed to despeckle SAR pictures

Wave propagation and transient response of a fluid-filled fgm cylinder with rigid core using the inverse Laplace Transform:

An examination on wave engendering and transient reaction of liquid filled Functionally Graded Material (FGM) is talked about by Daneshjou et al., 2017 [10]. Logical technique for inferring transient reaction oF liquid filled FGM round and hollow shell with a co-pivotal inflexible center. Converse Laplace transform is utilized for the investigation. Inference of wave engendering, transient reaction of liquid filled FGM chamber with unbending center utilizing transform procedure did.

Analysis of solutions of abel integral equations in astrophysics with the help of Laplace transformations

Kumar et al., 2015 [11] talked about a calculation for Abel essential equation, called as Homotopy Perturbation Transform method (HPTM). Relatively it discovered straightforward than other estimation techniques. The HPTM is utilized to get fast and precise arrangements of solitary basic equations of Abel type, linear and non-linear sort issues in science and innovation. Here the new strategy HPTM is shaped by some change of Laplace transformation.

\section{CONCLUSION}

Through this paper we have given brief information about Laplace and Inverse Laplace transformation and also provide applications of Laplace transformations in many fields .It is reviewed and explained that how Laplace transforms is used to sort-out the problems and equations not only in the field of mathematics but also in engineering, sciences, etc. Some applications of Laplace Transforms are reviewed and explained in an easy way. We have discussed ,how we can find the solutions to various complex and unique problems. The 
transformation of Laplace is therefore a key method in the study of circuits. Therefore, Laplace plays an important role in the field of engineering.

\section{REFERENCES}

1. Hailongchen, Yan zhang. [2015] Laplace Transform for stable random variables. IEEE 3rd international conference on computer and computing science.

2. Metwally IA. [1999] Simulation of impulse response of electric machines. IEEE transactions on energy conversion, 14: 861-867.

3. Hasan Modirshanechi, Naserpari. [2003] General nonlinear modal representation of large scale power systems. IEEE transactions on power systems, 18(3): 1103-1109.

4. Siriyaskolthanarat. [2007] Three level back-back HVDC based on H-bridge converter. Proc. IEEE Southeast conference.

5. Peter Lloyd woodfield. [2015] Transient analytical solution for motion of vibrating cylinder in the stokes regime using Laplace transforms. Journal of fluids and structures, 54: 202-214.

6. Szymczyk P, Szymczyk M. [2015] Classification of geological structure using ground penetration radar and Laplace transform artificial neural networks. Neurocomputing, 148: 354-362.

7. Shu-NanLi, Bing-Yang Cao. [2016] Generalisedvariational principles for heat conduction models based on Laplace transforms. International Journal of heat and mass transfer, 103: 1176-1180.

8. Mikail F. Lumentat. [2012] Analytical techniques for broadband multi electrochemical piezoelectric bimorph beams with multifrequency power harvesting. IEEE transactions on ultrasonics, ferroelectrics and frequency control, 59: 2555-2568.

9. Hazarika D, Nath VK, Bhuyan M. [2016] SAR imaging despecling based on combination of Laplace mixture distribution with local parameters and multiscale edge detection in lapped transformation domain. Procedia computer science, 87:140-147.

10. K. Daneshjou, M. Bakhtiari, A. Tarkashvand. [2017] Wave propagation and transient response of a fluid filled FGM cylinder with rigid core using inverse Laplace transform. European journal of mechanics A/ solids, 61: 420-432.

11. Sunil Kumar, Amit Kumar, Devendra Kumar, Jagev Singh, Arvind Singh. [2015] Analytical solution of abel integral equation arising in astrophysics via Laplace transform. Journal of Egyptian mathematical society, 23: 102-107. 